\title{
An Uni-directional Single-Phase Soft-switched Resonant High Frequency Link Inverter
}

\author{
Vishal Anand A G \\ Member, IEEE \\ Bloom Energy (I) Pvt Ltd \\ Bangalore, India \\ vanand@bloomenergy.com
}

\author{
Kaushik Basu \\ Member,IEEE \\ Indian Institute of Science \\ Bangalore, India \\ basu@ee.iisc.ac.in
}

\author{
Ranganathan Gurunathan \\ Member,IEEE \\ Bloom Energy (I) Pvt Ltd \\ Bangalore, India \\ rgurunat@bloomenergy.com
}

\begin{abstract}
Renewable and alternate energy grid integration is an area of interest in the recent years due to the advancements made in Solar and Fuel Cells. This paper considers a singlestage single phase uni-directional High-Frequency Transformer (HFT) link DC-AC converter for grid integration of Distributed Energy Resources (DER). The HFT helps by providing galvanic isolation to protect DER from single faults at significantly low cost and size in comparison with Low Frequency Transformer (LFT), Resonant based power conversion in relatively high voltage DC DC converters results in higher efficiency and power density Soft switching due to device capacitances and HFT parasitic leakage inductances enables high frequency of operation. This paper presents a novel Parallel Resonant Converter (PRC) based control strategy that results in high quality adjustable frequency and amplitude $\mathrm{AC}$ and soft switching of all devices in the entire range of operation. A well-known single stage isolated inverter topology is slightly modified to incorporate PRC based operation. Simulation results are presented to validate the topology and proposed modulation scheme.
\end{abstract}

\section{INTRODUCTION}

Power electronic converters and topologies which can integrate alternate sources like solar, wind, fuel cells etc. to the legacy grid are gaining a lot of traction due to the emphasis for clean electric power. When integrating DER into the grid, it's essential to maintain galvanic isolation to protect the capital equipment from single faults [1] and from the point of view of safety in some national codes [2]. The state of the art in isolated DC-AC is implemented with a High Frequency Link (HFL) DC-AC-DC followed by a hardswitched $\mathrm{H}$ bridge inverter. These two stages are connected through an electrolytic capacitor [3]. Single stage capacitor less bidirectional topologies usually employ four-quadrant switches in the AC side converter [4]. The control for this type of topologies can either be Pulse Width Modulated (PWM) [4] or dual active bridge based [5]. In [6] a series resonant based control is implemented. An indirect implementation of the $\mathrm{AC}$ side converter results in two $\mathrm{H}$-bridge inverters connected in cascaded configuration [7]. In [8], one of the bridge is converted into an uncontrolled rectifier and supports uni-directional power flow. The suggested control is PWM based and results in line frequency switching of the grid side inverter and partial soft-switching of the DC side $\mathrm{H}$ bridge. Resonant based power conversion is preferred due to 978-1-5386-9316-2/18/\$31.00 @ 2018 IEEE soft-switching and participation of high frequency transformer parasitics in the power transfer. In this paper a parallel resonant based control strategy is proposed for the topology given in [8] with a slight modification: Resonant capacitor $C_{r}$ is added as shown in Fig 1. The oscillation observed in PWM based control due to the device parasitic capacitors in the AC side converter is avoided here [9]. For low line condition duty cycle modulation is used. The proposed solution has following advantages 1) High quality adjustable magnitude and frequency output voltage synthesis 2) Line frequency lossless switching of the AC side bridge 3) Snubber-less softswitching of the DC side H-bridge 4) High power density due to HFL isolation. This paper provides the operation of the converter in steady state, hybrid modulation scheme and a step by step design procedure. Simulation results are provide for validation of the proposed topology.

\section{STEAdy STATE ANALYSIS}

This section presents the steady state analysis of the converter. The analysis is divided into the following sub-sections. (a) Variable frequency modulation (b) Hybrid modulation scheme and (c) Design methodology of the converter

\section{A. Variable Frequency Modulation}

The architecture of the proposed converter is shown in Fig 1. Before getting into the operation of the converter, the perunitization base quantities used for the purpose of analysis are defined in Table I.

TABLE I: Per-unitisation Base parameters

\begin{tabular}{|c|c|}
\hline Description & Formula \\
\hline Base Voltage, $V_{b}$ & $n V_{d c}$ \\
\hline Base Impedance, $R_{b}$ & $n \sqrt{\frac{L_{r}}{C_{r}}}$ \\
\hline Base Current, $I_{b}$ & $\frac{V_{b}}{R_{b}}$ \\
\hline Base Frequency, $f_{o}$ & $\frac{1}{2 \pi n \sqrt{L_{r} C_{r}}}$ \\
\hline
\end{tabular}

The High Frequency DC-AC (HF DC-AC) converter is modulated to generate a voltage at the output, $v_{o}$, such that it has a component at the grid frequency, $f_{g}$, with appropriate amplitude and phase shift with respect to the grid voltage, $v_{g}(t)=V_{g} \sin \left(\omega_{g} t\right)$ where, $\omega_{g}=2 \pi f_{g}$ as shown 


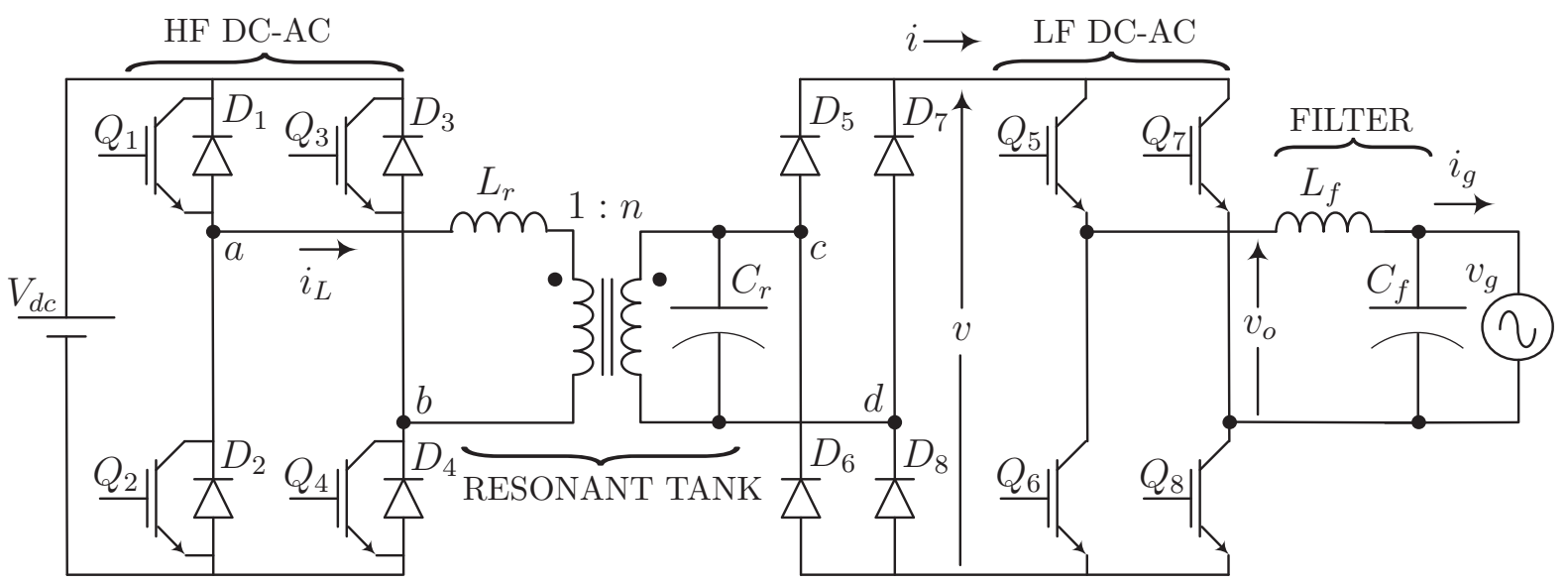

Fig. 1: Proposed topology for Grid integration of Distributed Energy Resources

in Fig 2(a). Let us define this component of $v_{o}$ to be the voltage, $v_{r e f}(t)=V_{o} \sin \left(\omega_{g} t+\phi\right)$. Given an active power reference $P^{*}$, the controller shall generate $v_{r e f}(t)$ by sensing $v_{g}(t)$. What follows is a description of the proposed resonance based Variable Frequency Modulation (VFM) to generate the grid frequency component of $v_{o}(t)$ following $v_{\text {ref }}(t)$.

Due to the filtering action of the inductor, $L_{f}$, we can assume that the line current, $i_{g}(t)$, is ripple free and purely sinusoidal at grid frequency. Due to the presence of the diode bridge, this current will be in phase with $v_{r e f}(t)$ as shown in Fig 2(a). So, $i_{g}(t)=I_{g} \sin \left(\omega_{g} t+\phi\right)$. The Per Unit (PU) grid current $J_{g}(t)=\frac{i_{g}(t)}{I_{b}}$. The current $i_{g}$ is shown in Fig 2(b). The AC side bridge is line frequency switched to rectify this current so that $J(t)=\frac{i(t)}{I_{b}}=\left|J_{g}(t)\right|$. The current, $i$ is also shown in Fig 2(b). The switching logic for switches $Q_{5}$ to $Q_{8}$ in the AC side converter (LF DC-AC) is also shown in Fig 2(b). The HF DC-AC bridge is switched to generate a square wave with frequency $f_{s}$ and amplitude $V_{d c}$ and applies it to the resonant tank as shown in Fig 2(a) as $v_{a b}$. The PU switching frequency is given by

$$
F=\left(\frac{f_{s}}{f_{o}}\right)=\left(\frac{\omega_{s}}{\omega_{o}}\right)
$$

where, $f_{s}$ is the HF DC-AC switching frequency, $\omega_{s}$ is HF DC$\mathrm{AC}$ angular switching frequency and $\omega_{0}$ is the angular base frequency. The tank responds with a quasi-sinusoidal voltage at switching frequency shown as $v_{c d}$ in Fig 2(b) and is rectified by the diode bridge $D_{5}$ to $D_{8}$ to get voltage waveform, $v$. The switching frequency average of the voltage across the diode bridge, $\bar{v}=M(t) n V_{d c}$ is a function of the normalized current, $J(t)$, and $F$ where, $M(t)$ is the gain of the resonant tank. The functional relationship for $M$ is given below assuming operation in Continuous Capacitor Voltage Mode (CCVM) above resonant frequency, $F>1$. The derivation of (2) is given in [10].

$$
M=\left(\frac{2 F}{\pi}\right)\left(\theta-\frac{\sin \theta}{\cos \frac{\pi}{2 F}}\right)
$$

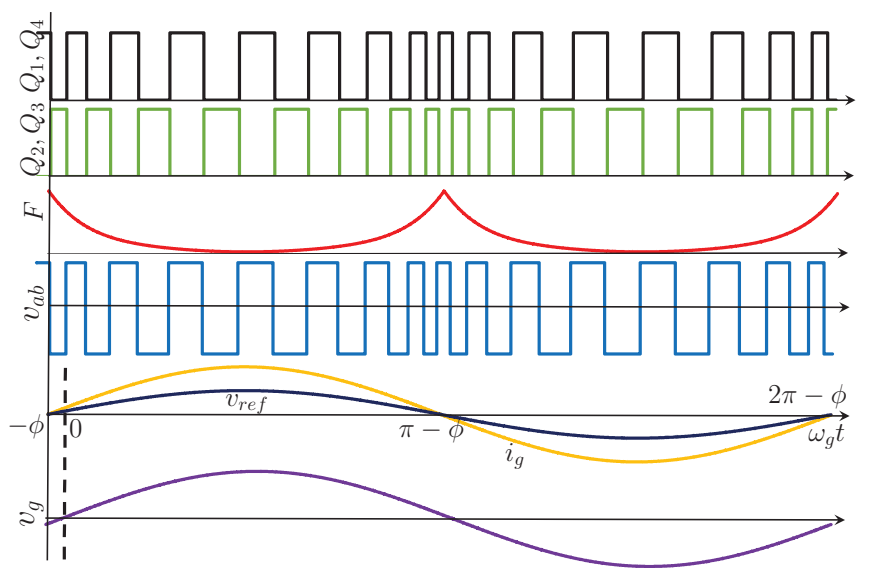

(a)

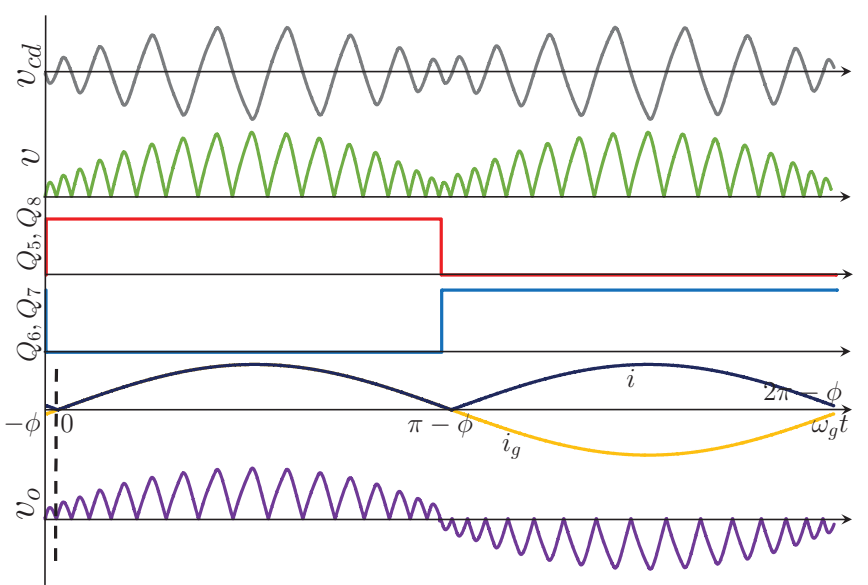

(b)

Fig. 2: Modulation of the Single stage HFL Inverter (a) High frequency modulation and key waveforms in the primary side converter (HF DC-AC) and (b) Line frequency modulation and key waveforms in the secondary side converter (LF DC-AC) 


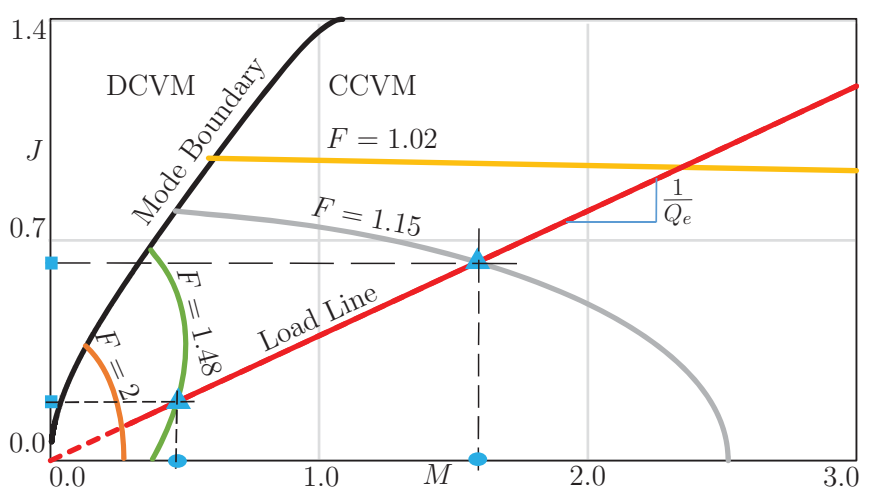

(a)

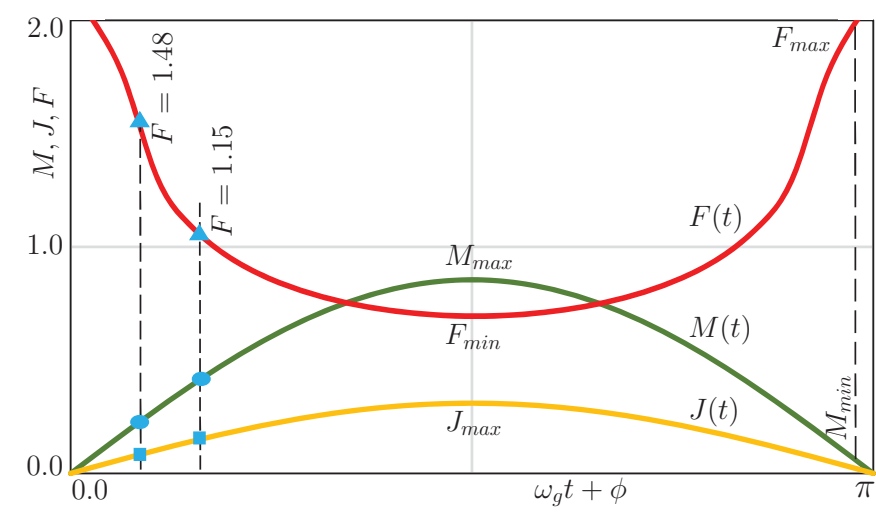

(b)

Fig. 3: Control Characteristics of the PR converter (a) Output Plane of the PR Converter under different frequencies of operation and (b) Time variation of the Switching frequency, Gain of the converter and DC Link Current over one fundamental half-cycle

$$
\theta=-\cos ^{-1}\left(\cos \frac{\pi}{2 F}+J \sin \frac{\pi}{2 F}\right)
$$

The switching frequency, $F$ is varied in time over the line cycle so that the switching frequency average component of $v(t)$ is $\left|v_{r e f}(t)\right|$. This variation in $F$ is shown in Fig 2(a). In turn, the fundamental component of $v_{o}(t)$ follows $v_{r e f}(t)$. This is also shown in Fig 2(a). As the switching frequency is varied over a fundamental line cycle, the proposed modulation strategy is termed as Variable Frequency Modulation.

The output characteristics of the PRC [10], as shown in Fig 3(a) plots the PU link current, $J$ as a function of PU voltage conversion ratio, $M$ for different values of PU switching frequency, $F$. The plots correspond to PU frequency in the range, $1<F<2$. Here operation above resonance, $F>1$ is considered, as this condition guarantees Zero Voltage Switching (ZVS) of the DC side bridge (HF DCAC) for all operating conditions. The curve corresponding to the "Mode Boundary" between CCVM and Discontinuous Capacitor Voltage Mode (DCVM) is also shown in this figure. As discussed earlier, $M(t)$ and $J(t)$ are in same phase as shown in Fig 3(b). For a given reference output power, the ratio between them, also known as "Quality Factor", $Q_{e}=\frac{M(t)}{J(t)}$ is independent of time. So, the operating point on the $M-J$ plane shall trace a straight line. This is shown in Fig 3(a) as "Load Line". The intersection of the load line on the output characteristics shall correspond to a particular operating point in the time-domain waveform shown in Fig 3(b) ( $F=1.48, F=1.15)$. Through design, we need to ensure that this line lies in the CCVM region of the $M-J$ plane. It's possible to see from Fig 3(b) that the frequency, $F$ reduces as the voltage conversion ratio, $M$ increases. So, when $M$ reaches $M_{\max }, F$ shall reach its minimum value, $F_{\min }$. In the reverse direction, as $M$ reduces, $F$ increases and reaches its maximum value $F_{\max }=2$ at a certain $M_{\min }$ as shown in Fig 3(b). As the steady state operation of PRC is not known beyond $F=2$, the maximum switching frequency is limited to twice the resonant frequency [10].

\section{B. Hybrid Modulation Scheme}

The output characteristics indicate that the maximum frequency of operation possible with the PRC is $F_{\max }=2$. As the maximum $F$ is limited to 2, to trace the dotted portion of the load line in Fig 3(a), constant frequency PWM is employed. For operation in this range, HF DC-AC bridge is pulse width modulated to generate a quasi-square wave at constant frequency of $F=2$. This is shown in Fig 4(a). The duty cycle of this waveform is adjusted to obtain necessary voltage gain and follow the reference voltage waveform, $v_{\text {ref }}(t)$. The soft switching of the converter is not guaranteed in this range of operation. As the HF DC-AC is modulated partly with variable frequency and rest of the time with variable pulse width as shown in Fig 4(b), the proposed modulation technique is termed as Hybrid Modulation Scheme (HMS).

Fig 5 shows the equivalent circuit of the single stage HFL

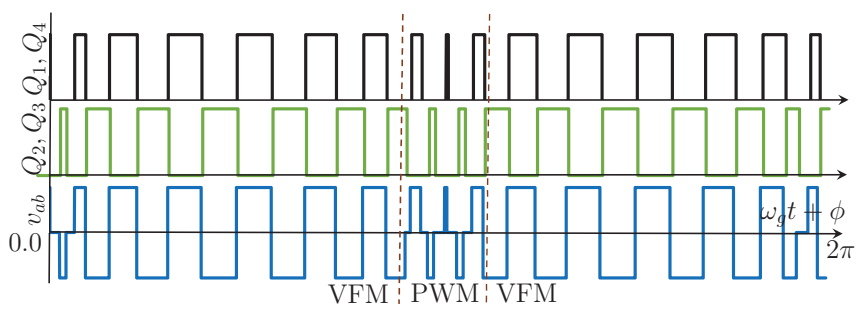

(a)

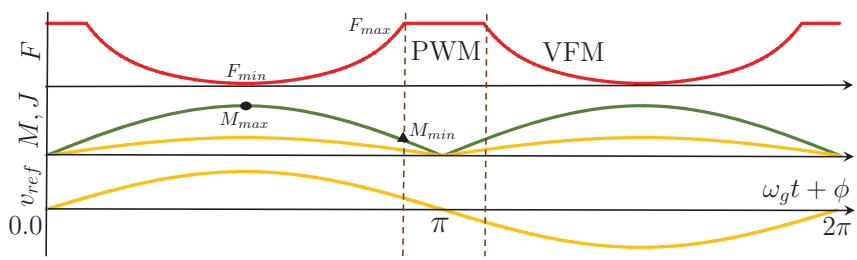

(b)

Fig. 4: Hybrid Modulation of the PR converter (a) VFM and PWM operations on the HF DC-AC and (b) Time variation of the Switching frequency, Gain of the converter and the voltage reference against which the modes are varied 


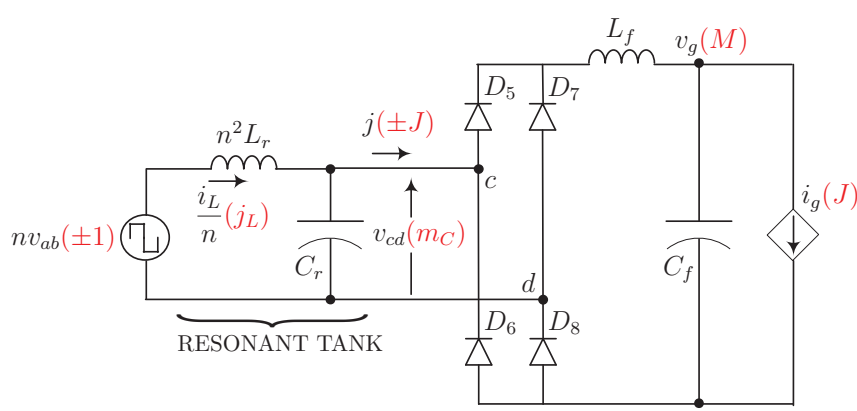

Fig. 5: Equivalent circuit of the Single Stage HFL Inverter for analysis

inverter for the purpose of circuit analysis reflected on the secondary side of the transformer. The analysis is performed for switching frequency circuit variations. Since this frequency is much higher than the fundamental line frequency, DC operating point analysis shall be performed going forward. The switching source, $n v_{a b}$ in Fig 5 represents the switching source driving the resonant tank circuit. The load is modelled as a voltage dependent current source, $i_{g}$ indicated as $J$ in PU due to the diode bridge on the secondary. This equivalent circuit and the related parameters based on the per-unitisation defined in Table I is shown in Fig 5. The per-unitised converter parameters based on the base quantities is given in Table II.

In the VFM mode of operation, based on the polarity of

TABLE II: Per-unitised quantities

\begin{tabular}{|c|c|}
\hline Description & Formula \\
\hline PU capacitor voltage, $m_{C}$ & $\frac{v_{c d}}{V_{b}}$ \\
\hline PU resonant inductor current, $j_{L}$ & $\frac{i_{L}}{I_{b}}$ \\
\hline Angular half-period, $\gamma$ & $\frac{\pi}{F}$ \\
\hline
\end{tabular}

the applied square wave impulse, $n v_{a b}$ and the polarity of the capacitor voltage, $m_{C}$ shown in Fig 5, the PRC can be reduced to 4 modes of operation [10]. In the PWM mode of operation, 2 additional modes are introduced due to the introduction of zero state in $v_{a b}$ waveform as shown in Fig 4(a). The steady state waveforms at a particular operating point under the PWM mode of operation are shown in Fig 6. Modes 1, 2 and 5 are observed when the applied voltage is $n v_{a b}$ or $+1 p u$. The behavior of the circuit in the other 3 modes is identical except that the initial conditions are complementary of modes 1, 2 and 5. The conducting devices during each of the modes is shown in Fig 6. The governing equations of the converter under different operating modes can be summarized as given below

$$
\frac{d j_{L}}{d \theta}+m_{C}=n v_{a b}
$$

where, $n v_{a b}=0, \pm 1$

$$
\frac{d m_{C}}{d \theta} \pm j=j_{L}
$$

where, $j= \begin{cases}+J, & \text { if } m_{C}>0 \\ -J, & \text { if } m_{C}<0\end{cases}$

These are the equations at a particular DC operating point.

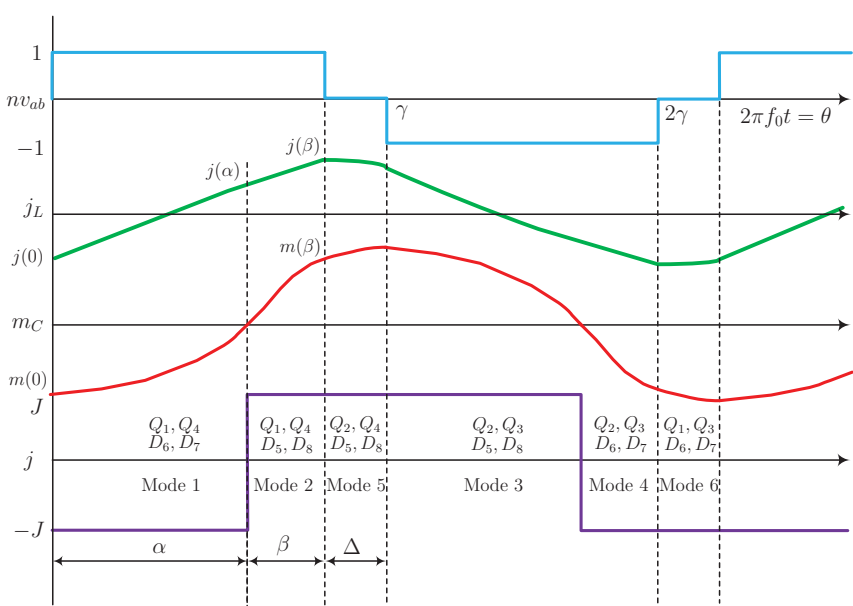

Fig. 6: Steady state waveforms of the parallel resonant converter at a particular operating point in PWM mode of operation

Over a fundamental line cycle, these points move as per the time variation given in Fig 3(b). In order to arrive at a closed form expression for the converter gain under the PWM mode of operation, state plane portrait of the resonant tank state variables, $j_{L}$ and $m_{C}$ is used as shown in Fig 7. Basic trigonometric concepts are used to eliminate common terms involving the conduction angles, $\alpha, \beta$ and $\Delta$. The expression for the converter gain in the PWM mode is given below

$$
M=\frac{2 F}{\pi}\left[\theta+\frac{\frac{\sin \Delta}{2}-\left(\sin \left(\theta-\frac{\Delta}{2}\right)\left(\cos \frac{\pi}{2 F}+J \sin \frac{\pi}{2 F}\right)\right)}{\cos \frac{\pi}{2 F} \cos \left(\theta-\frac{\Delta}{2}\right)}\right]
$$

where, $\theta$ is defined in (3). One observation that can be made from (6) is that as $\Delta$ tends to 0 in the VFM mode of operation, the converter gain, $M$ shall become equal to the value derived in (2).

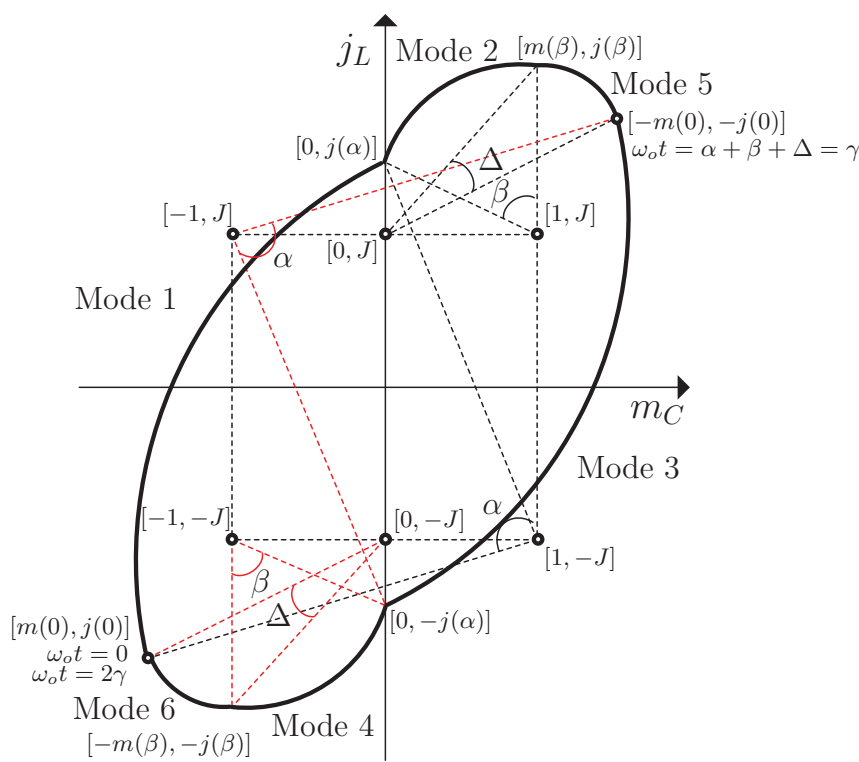

Fig. 7: State plane portrait of the PRC HF DC-AC in PWM mode of operation 


\section{Design And Simulation Results}

The following sections delve into the design steps of the converter to ensure converter operation and achieve ZVS over the entire range and the selection of the components. Simulation results are provided to validate the topology.

\section{A. Design Steps}

The design is based on the specifications given in Table III. Design implies determination of the values of $L_{r}, C_{r}$ and

TABLE III: Converter Design specifications

\begin{tabular}{|c|c|}
\hline Description & Value \\
\hline Input Voltage $V_{d c}$ & $390 \mathrm{~V}$ \\
\hline Grid Voltage RMS $V_{g, r m s}$ & $230 \mathrm{~V}$ \\
\hline Grid Frequency $f_{g}$ & $50 \mathrm{~Hz}$ \\
\hline Power Output $P_{o}$ & $2 \mathrm{~kW}$ \\
\hline Max Switching Freq $f_{s, \max }$ & $100 \mathrm{kHz}$ \\
\hline
\end{tabular}

turns ratio, $n$ in Fig 1. This is same as choosing $f_{o}, J_{\max }$ and $M_{\max }$. As we know $f_{s, \max }=2 f_{o}$ and from the design specification, $f_{s, \max }=100 \mathrm{kHz}$, the resonant frequency $f_{o}$ will be $50 \mathrm{kHz}$. As discussed earlier that at rated condition the operating point traces a straight line, passing through the origin, in the $M-J$ plane. This line is labeled as "Load Line" in Fig 3(a). For lower active power transfer the trajectory shall be another straight line but with a smaller slope. As the grid voltage remains constant (so is the output voltage as the drop across $L_{f}$ at grid frequency is small), $M_{\max }$ will not change significantly. So, for all other loading conditions, the operating point will be inside the shaded triangle as shown in Fig 8 . The design is complete once we choose the tip of this triangle $\left(M_{\max }, J_{\max }\right)$. For operation in CCVM and above resonance,

this triangle must lie in a region bounded by a) the horizontal line $J=1$ (this is also the $M-J$ contour corresponding to $F=1$ as the PRC behaves as a current source at resonant frequency) and b) the "Mode Boundary" curve that can be approximated with a straight line, $J_{\text {crit }}=1.25 * M+0.21$ as shown in Fig 8. So the point $\left(M_{\max }, J_{\max }\right)$, must be in this region. This point is selected based on considerations of conduction loss or RMS Inductor current and sensitivity of gain variation $M$, towards the peak, $M_{\max }$ with respect to $F$. This choice also decides $M_{\min }$. The values of $M_{\max }=1.0$ and $J_{\max }=0.9$ are chosen. The calculated values of other

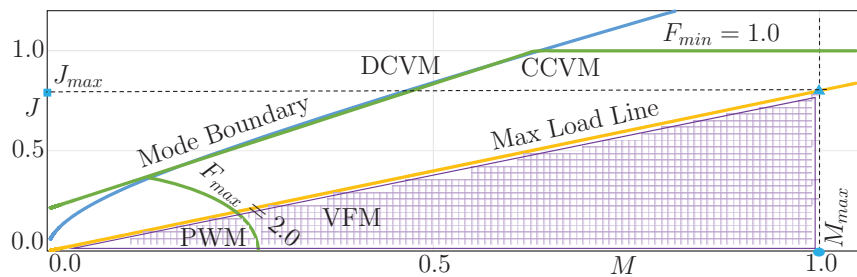

Fig. 8: Design of the Parallel Resonant converter based on the choice of $J_{\max }$ and $M_{\max }$ in the Output Plane components are $L_{r}=108.9 \mu H, C_{r}=78.2 n F, n=0.917$. The choice of $L_{f}$ and $C_{f}$ are done based on the filtering requirement of the high frequency component and acceptable line impedance drop in the filter inductance. The values of $L_{f}=2.53 \mathrm{mH}$ and $C_{f}=6.37 \mu \mathrm{F}$ are finalized for the design.

\section{B. Simulation Results}

The converter is simulated with the proposed modulation strategy and circuit parameters obtained through design at the rated operating conditions given in Table III. Fig 9(a) shows the grid current, $i_{g}$ along with the grid voltage, $v_{g}$. They are almost in the same phase as the voltage drop across $L_{f}$ is negligible. Fig 9(b) shows the Output voltage waveform, $v_{o}$ that has an envelope of the reference voltage waveform at the grid frequency. Fig 9(c) presents the spectrum of this voltage confirming high quality output voltage generation. The waveform of tank voltage, $v_{c d}$ in Fig 10(a) shows the low frequency envelope. A zoom into this waveform as given in Fig 10(b) indicates the high frequency switching component.

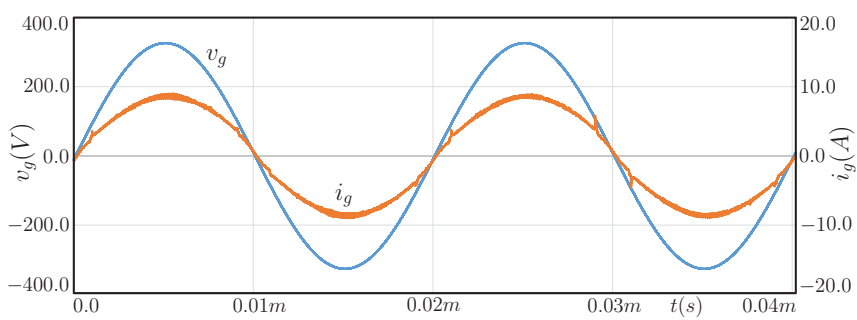

(a)

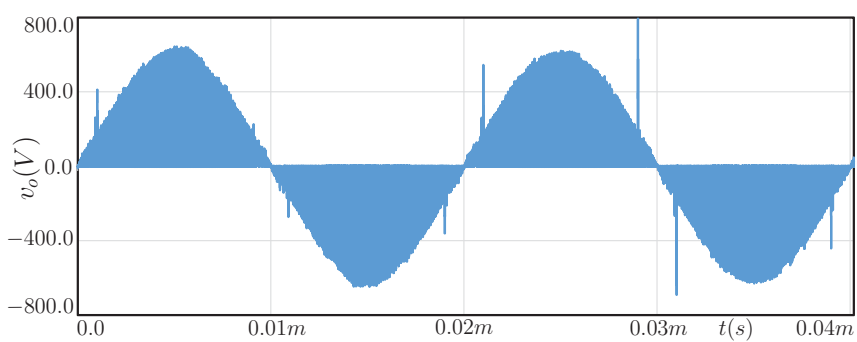

(b)

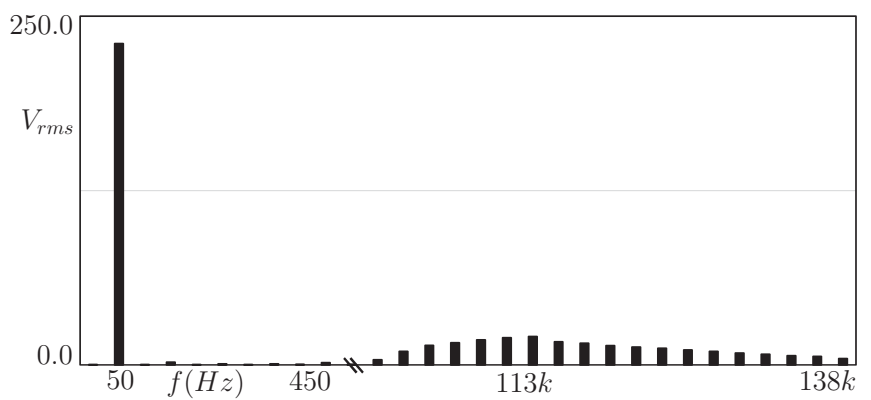

(c)

Fig. 9: Simulation of the PRC based Single Stage Single phase HFL Inverter (a) Waveform of Grid Voltage, $v_{g}$ and Current, $i_{g}$, (b) Waveform of the LF DC-AC Output Voltage, $v_{o}$ and (c) FFT of the Output Voltage waveform, $v_{o}$ indicating the various frequency components 


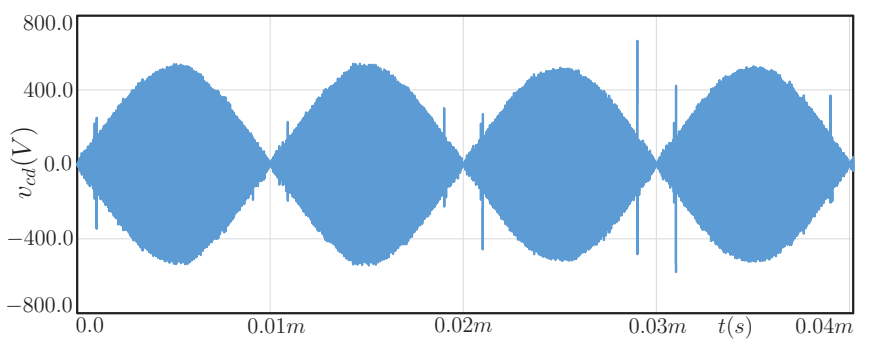

(a)

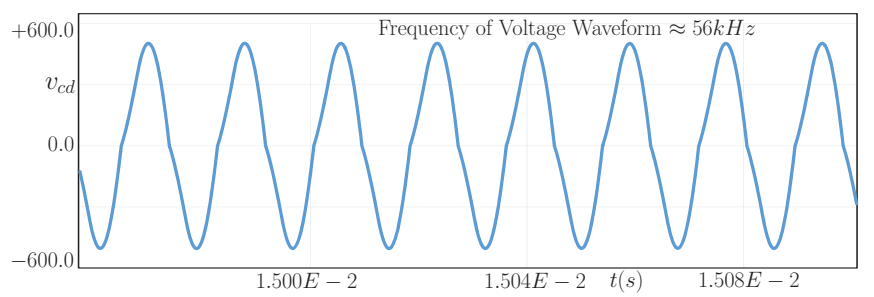

(b)

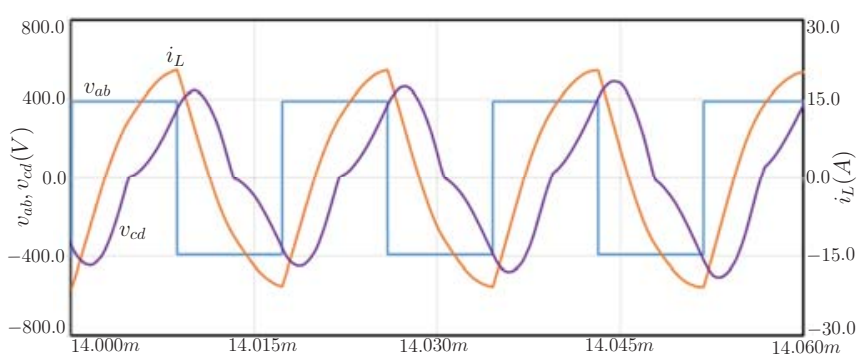

(c)

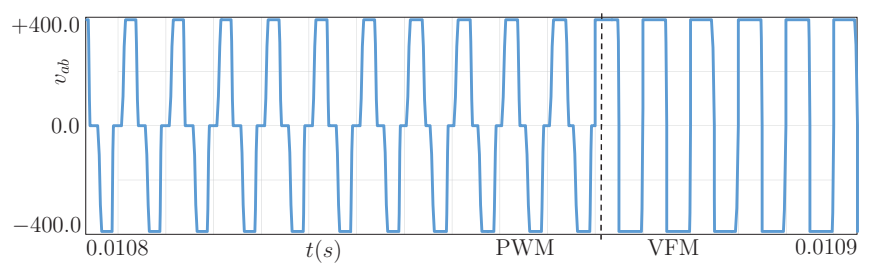

(d)

Fig. 10: Simulation of the PRC based Single Stage Single phase HFL Inverter (a) Waveform of capacitor voltage, $v_{c d}$ indicating line frequency bi-polar envelope, (b) Zoom into the waveform of capacitor voltage, $v_{c d}$ indicating the high frequency switching component (c) Waveforms of applied square wave, $v_{a b}$, resonant inductor current, $i_{L}$ and capacitor voltage, $v_{c d}$ and (d) Waveforms of applied square wave, $v_{a b}$ showing the boundary between PWM and VFM modes of operation

These waveforms are in-line with the prediction of the converter operation as described in Fig 2(b). The HF DC-AC bridge output voltage and HFT primary current as shown in Fig 10(c) confirm ZVS operation. The waveform shown in Fig 10(d) indicates the changeover from the PWM mode to the VFM mode of operation validating the hybrid modulation scheme.

\section{CONCLUSION}

In this paper, a single phase single-stage high frequency link soft-switched uni-directional inverter is proposed. The HFT in this architecture provides the isolation and high power density for design. This topology can be used for grid integration of solar and fuel cells modules. Power transfer from DC to AC is based on parallel resonant control on the primary side devices. ZVS over most of the operating range on the primary bridge and low frequency switching on the AC side converter ensure high efficiency of operation. Variable frequency modulation along-with hybrid modulation scheme is described in detail and the design steps have been explained for selection of circuit components. Simulation has been performed to validate the proposed topology and results have been presented which are in line with the analysis.

\section{REFERENCES}

[1] ABB. (2011) Pv inverter designs and operation. [Online]. Available: http://www.iaeisj.org/sitebuildercontent/sitebuilderfiles/abb_ pv_inverter_designs_and_operation_rca16sep2014abb.pdf

[2] S. Kouro, J. I. Leon, D. Vinnikov, and L. G. Franquelo, "Grid-connected photovoltaic systems: An overview of recent research and emerging pv converter technology," IEEE Industrial Electronics Magazine, vol. 9, no. 1, pp. 47-61, March 2015.

[3] S. Inoue and H. Akagi, "A bidirectional dc-dc converter for an energy storage system with galvanic isolation," IEEE Transactions on Power Electronics, vol. 22, no. 6, pp. 2299-2306, Nov 2007.

[4] N. Kummari, S. Chakraborty, and S. Chattopadhyay, "An isolated highfrequency link microinverter operated with secondary-side modulation for efficiency improvement," IEEE Transactions on Power Electronics, vol. 33, no. 3, pp. 2187-2200, March 2018.

[5] R. Baranwal, G. F. Castelino, K. Iyer, K. Basu, and N. Mohan, "A dualactive-bridge-based single-phase ac to dc power electronic transformer with advanced features," IEEE Transactions on Power Electronics, vol. 33, no. 1, pp. 313-331, Jan 2018.

[6] S. N. Vaishnav and H. Krishnaswami, "Single-stage isolated bidirectional converter topology using high frequency ac link for charging and v2g applications of phev," in 2011 IEEE Vehicle Power and Propulsion Conference, Sept 2011, pp. 1-4.

[7] A. Kulkarni and V. John, "New start-up scheme for hf transformer link photovoltaic inverter," IEEE Transactions on Industry Applications, vol. 53, no. 1, pp. 232-241, Jan 2017.

[8] A. Pal and K. Basu, "A bidirectional snubber less soft-switched high frequency link dc/ac converter," in 2016 7th India International Conference on Power Electronics (IICPE), Nov 2016, pp. 1-8.

[9] G. M. K. Park, C. Kim and M. Youn, "Voltage oscillation reduction technique for phase-shift full-bridge converter," IEEE Transactions on Industrial Electronics, vol. 54, no. 5, pp. 2779-2790, Oct 2007.

[10] S. D. Johnson and R. W. Erickson, "Steady-state analysis and design of the parallel resonant converter," IEEE Transactions on Power Electronics, vol. 3, no. 1, pp. 93-104, Jan 1988. 